\title{
Wireless Communication using VLC and MIMO Technology
}

\author{
Dr. Nitesh Gupta
}

\author{
Associate Professor and HoD \\ Department of Computer Science Engineering \\ Technocrats Institute of Technology, Bhopal, India.
}

\begin{abstract}
Due to the rapid growths in Light Emitting Diodes, VLC (Visible Light Communication) has received a great attention in last years. Our main aim of this research work is to build the wireless VLC (Visible Light Communication) system which should be able to transmit the data in-between two computers with the use of a visible light. We are using an LED array as the transmitter, Light Dependent Resistor (LDR) as the receiver component and air as the transmission medium. In both the transmitter and receiver modules, we are using Arduino UNO microcontroller board. Two computers have been used to interface the software to the hardware with the help of Arduino IDE installed to both of them. If we want to check the performance of VLC system with MIMO, commercial optical system simulation software called OptiSystem7.0 from Optiwave can be used. The layout diagram for both SISO (Single Input Single Output) and MIMO (Multiple Input Multiple Output) communication has been generated and has been run and the BER (Bit Error Rate) performance is being analyzed. FSO (Free Space Optical) communication channel is used as transmission medium for both SISO and MIMO. Data transmission and reception for 100 meters is done using OptiSystem software. The eye diagram of BER analyzer showed that the maximum quality factor of MIMO is relatively large when compared to SISO.
\end{abstract}

Index Terms: SISO (Single Input Single Output), Visible Light Communication (VLC), Light Emitting Diode (LED), Arduino, MIMO (Multiple Input Multiple Output).

\section{INTRODUCTION}

In the last decade, the demand for data usage has increased exponentially. All the peoples want to be connected to internet whole time, on multiple devices at the same time, update the latest happenings at each place etc. With the advancement of IOT (Internet of Things) more and more devices will get connected to the LTE which will result in speed decrement and congestion. Considered multiple options and of them was to utilize the unused visible light spectrum to solve this crisis and it risen the new concept called Light fidelity ( $\mathrm{Li}-\mathrm{Fi})$. As the meaning of those terms are one and the same. Li-Fi is the kind of visible light communication structure. VLC is also a part of the Optical Wireless Communication. These techniques can enable the communication by modulating the light in the visible spectrum, which can mostly be used for the illumination resolves [1]. Li-Fi is unique because here the same light is being used for communication purpose and also illumination. Radio wave spectrum is very limited and visible light spectrum is made use for the communication determination.

Visible light communication, as the name says it is a communication technique based on visible light. This spectrum is a part of electromagnetic spectrum and that is surely visible to the human eye. The visible light in common terms is simply called as light itself. This mode of communication is wireless. Nowadays, IR (Infrared) systems have been developed to provide more data rate, because the conventional systems such as Bluetooth, radio communication and Wi-Fi had low speed. VLC system uses different colored LED's and some colored LED combinations to produce white light. Some filters will be used in the receiver side to separate different colored lights. White LED's are the most widely used ones in visible light communication system. In order to achieve high data rate in our hardware, we made use of array of LED's. The array of LED's provides better illumination as well as to achieve peak data rate. New modulation and multiplexing techniques are developed and used by the researchers to provide high data rate. LED flashlights and lasers are used in some applications which require high intensity light for long distance communication [1].

Taking Li-Fi as a consideration, it is a type of visible light communication method. It offers unlicensed spectrum and thus users can utilize wider range of bandwidth. Visible light is very much harmless to use, as any health hazards will not happen through it. This is because visible light don't penetrate through the wall, whereas electromagnetic waves can easily penetrate through the walls. Wi-Fi is not permissible in some of the hospitals due to harmful radiations. The direct line of sight (LOS) is one of the basic requirements for any kind of visible light communication. LOS between transmitter and receiver should be proper to achieve better data rate. If any obstacle/object or undesired wave come in between the transmitter and receiver, at that time the line of the sight gets disturbed and it cause denial of service (DoS). But the major advantage of using visible light spectrum is that, any hacker or intruder outside the room will not be able to utilize data. Because of this reason more secure communication takes place [2].

SISO (Single Input Single Output) systems are most of the times used for smaller applications. Adding MIMO (Multiple Input Multiple Output) technology along with VLC is a better idea for larger applications in real time. In this paper we have designed a hardware setup for PC to PC communication using VLC. This is a SISO system and an experimental setup for smaller distance. This can be further improved if high intensity light sources are used. And to check the advantages of including MIMO in VLC system, we did some simulations. The BER (Bit Error Rate) performance of both SISO and MIMO is analyzed. As this is indoor VLC application, distance between transmitter and receiver is set in terms of meters. In case of outdoor scenario, distance can be 
increased up to kilometer range. But the FSO (Free Space Optical) channel should be used and atmospheric conditions must be taken into consideration.

\section{LITERATURE REVIEW}

Some papers which are related to visible light communication, MIMO, LED, free space optics, etc are referred for this project work. Satish R. Devane \& Zashi P. Choudhari have designed high sensitive universal Li-fi receiver. The main aim was to enhance the data communication. In this research paper, authors describes the working and characteristic features of LED based visible light communication. They showcase the advantages of using non-hazardous LED light [3]. Shaik.Shakeera et.al designed a Computer to Computer File Transfer system with the use of Li-Fi Technology. The main aim of their work was about text data transmission. Text transmission from PC to PC is executed with better speed than Wi-Fi. Their work is an inspiration to develop hardware model for LiFi. In future scope, authors highlighted about Li-Fi hotspots, which can be transmitted in Gbps order [4].

Akash Gupta et.al developed a Cascaded FSO-VLC Communication System. Both VLC and FSO are the major areas of interest in our work. The performance of FSO-VLC system is being analyzed by them. Different indoor and outdoor parameters are considered in their work. Their work provides a solution to spectral congestion problem faced by most of the wireless communication types [5]. Chetna Verma and Chetan Selwal used diversity technique invisible light communication system. Along with that they included 4 QAM (Quadrature Amplitude Modulation) OFDM (Orthogonal Frequency Division Multiplexing) FSO (Free Space Optical) link. Authors simulated utilizing OPTISYSTEM 12 version. They designed 4QAM OFDM FSO link in three different stages. And also the performance of the system for different environmental conditions was simulated and analyzed [6].

Hammed G. Olanrewaju et.al illustrated MIMO-OFDM system in their paper. The BER plots obtained in their simulation show that high performance gains are achieved by using DPWC (Dual Pair-wise Coding) [7]. Fabian Harendran Jesuthasan et.al designed SISO-VLC and 2x2 MIMO setup. They transmitted the audio sound through both the setups. They concluded that quality and receiving range was improved in MIMO-VLC when compared to SISO-VLC system [8].

\section{HARDWARE MODEL}

The hardware model consists of two PC's (Personal Computers) at both transmitter and receiver side. And also two Arduino UNO boards are required at the transmitter and receiver modules. Arduino UNO is the hardware which is fully based on the microcontroller ATmega 328P. It is having 14 digital input/output pins out of which, we can use 6 as PWM outputs, 6 as analog inputs, a $16 \mathrm{MHz}$ quartz crystal, a USB connection, a power jack, a reset button and an ICSP header [9]. LED array is used as source of illumination and as data transmitter [10]. LED arrays are assemblies of LED packages that provide high intensity light. The white LED's are being used in this hardware model. TIP122 is a bipolar transistor and is used at the transmitter side circuit. The main thing to remember about this transistor is that it allows a current of $5 \mathrm{~A}$ to flow from the emitter through the collector and $120 \mathrm{~mA}$ from the emitter through the base to flow [11]. The hardware is shown in Fig.1.

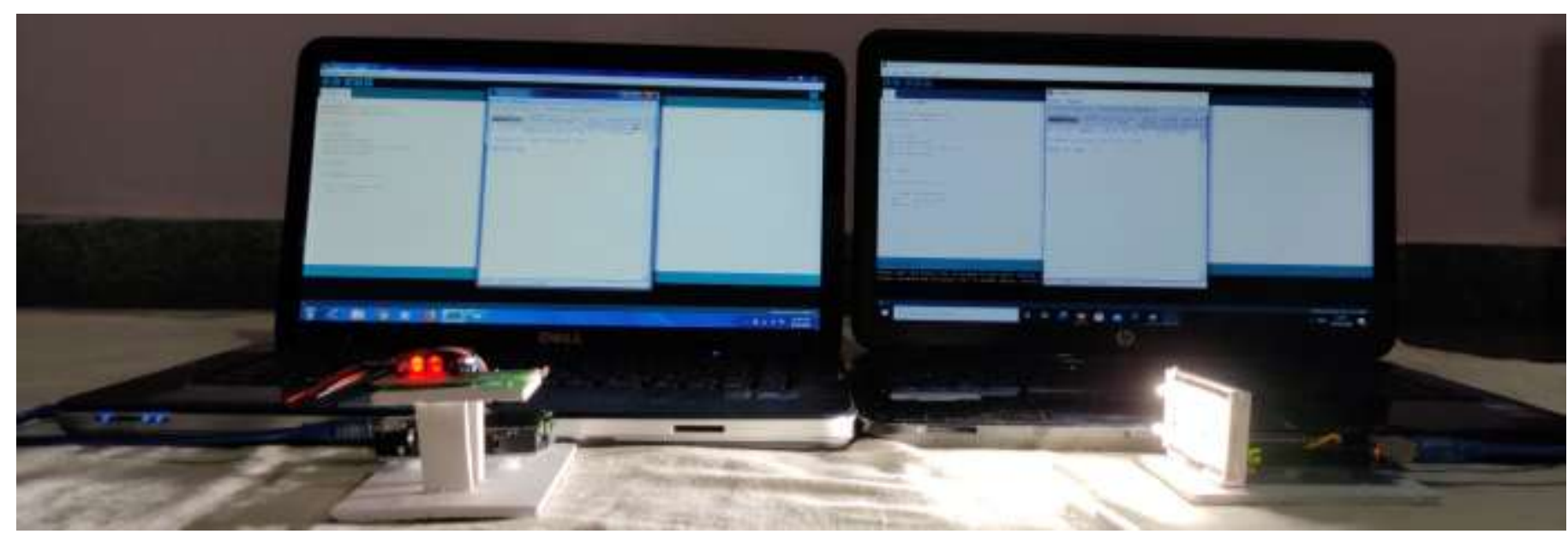

Fig.1: The hardware of $\mathrm{PC}$ to $\mathrm{PC}$ communication transmitter and receiver

Coming to the receiver side there is a LDR Sensor Module which can be used if we want to detect the presence of light or if we want to measure the intensity of light. The output of this module goes to high level whenever the presence of light is there and it also goes to low level whenever the absence of light is there [12]. A single LDR module will be used and can detect the light for around 15 centimeter distance. The Arduino IDE and X-CTU software (serial terminal software) was installed in both PC's. The embedded C programming is done on Arduino IDE of both transmitter and receiver side and are compiled. Once both transmitter and receiver side codes are compiled without error. The programs are then dumped into Arduino UNO boards after connecting the PC using USB connector. Once connections and program dumping is over, the message is typed on the serial monitor of transmitter side PC. The same message will be displayed on the receiver side PC's serial monitor. The text transmission was successful for smaller distance that is around 15 centimeters. To further check the performance of Visible light communication for larger distance and for real time applications, layout design and simulations are done using OptiSystem software. 


\section{Simulation SYSTEM MODEL}

In this proposed system, commercial optical system simulation software (OptiSystem7.0) from Optiwave is used. The layout diagram for both of Multiple Input Multiple Output \& Single Input Single Output have been generated and has been run and the BER performance is being analyzed. A CW (Continuous Wave) laser diode is used at transmitter side with $1 \mathrm{~W}$ power and visible light frequency. A CW laser with frequency of red color is used, that is $700 \mathrm{~nm}$. A pseudo random bit sequence generator is used for data bits generation which supports a high data rate of about $35 \mathrm{G}$ bits/second. Both data bits and laser light will be fed into a Mach-Zehnder modulator. This MZ modulator is used for controlling the amplitude of an optical wave. Optical amplifier is used to increase the signal strength. FSO (Free Space Optical) Channel is used as communication channel. This FSO is not only meant for indoor communication, it is suitable for free space communication as well [13]. FSO is a form of optical communication technology where larger distances can be covered [14]. I have done data transmission and reception for 100 meters. But it is even possible to communicate in terms of kilometers. As this project is based on indoor VLC, a 100 meter distance is more than enough for indoor applications.

At the receiver side, Avalanche photodiode is being used. It is extremely sensitive semiconductor photodiode that feats photoelectric effect to covert light into an electricity. For long range communication, this APD is very much suitable when compared to other diodes [15]. The Bessel filter is enhanced to offer a constant group delay in the filter pass-band, while forfeiting intelligence in the magnitude reply. 3R regenerator is a subsystem based on the Data Recovery component and a NRZ (Non Return to Zero) pulse generator. Instead of connecting the bit error rate analyzer to input signals, this component $3 \mathrm{R}$ regenerator can be used to recover the bit sequence. The reference signal is nothing but the output signal itself [16]. Electrical and optical power meters are connected to some components to check the power dissipation over distance.

A general modulation format which can be useful in optical communication is Non-return to zero. It is also known as OOK (OnOff Keying) because during any communication, the light source will be switch on and off and it depends on logical value of binary data signal. The logged trace can be disjointed into 1.5 or 2 bit segments and then they are covered on the top of each other so that it will form an eye diagram. For signal deficiency detection in OOK signaling we can also use eye diagram. BER analyzer is used at the receiver to know the BER performance [17]. As both SISO and MIMO layouts are designs. BER performance of MIMO over SISO is compared. The layout diagram of SISO VLC is as shown in Fig.2. A single transmitter and single receiver with one FSO channel are used in layout. The BER analyzer can automatically calculate the bit error rate and a Quality factor.

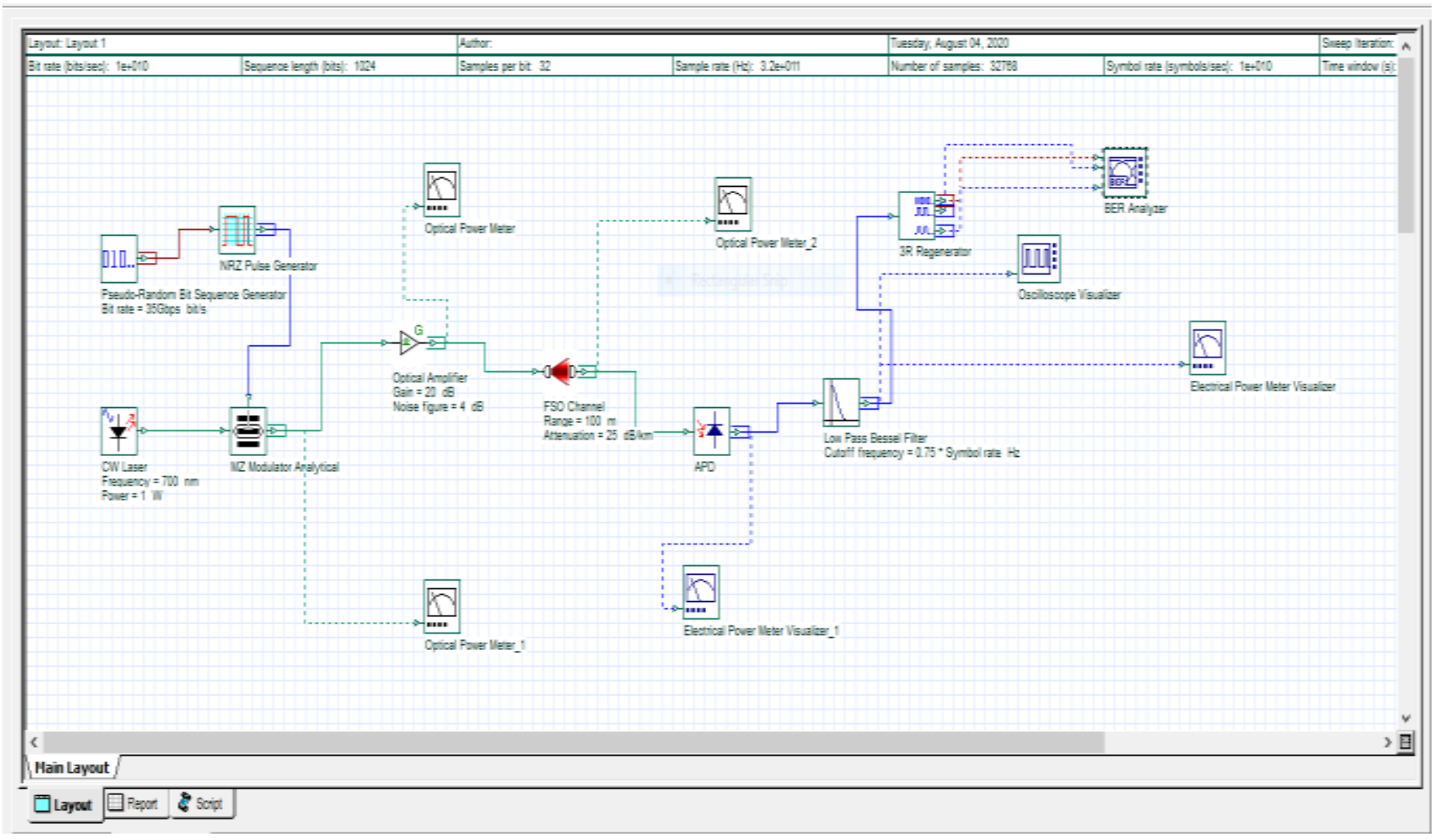

Fig.2: The layout of SISO (Single Input Single Output) Visible light communication transceivers using FSO channel.

MIMO takes benefit from the spatial multiplicity and it receives multiple autonomous copies of same signal at the receiver side. MIMO system will increase signal to noise ratio \& BER [8]. The optical amplifier power is increased in the layout design, as we have to transmit more power. The MIMO technology can also increase data rate. And it is also capable of improving the system dependability through spatial multiplicity [7]. In the layout of MIMO transceiver, four transmitters and receivers are used. A 1 to $\mathrm{N}$ fork is used to multiply the number of transmitters. And power combiner is used at the receiver side to multiply the receiver blocks. The layout designed for MIMO VLC system using OptiSystem software is shown in Fig.3 below: 


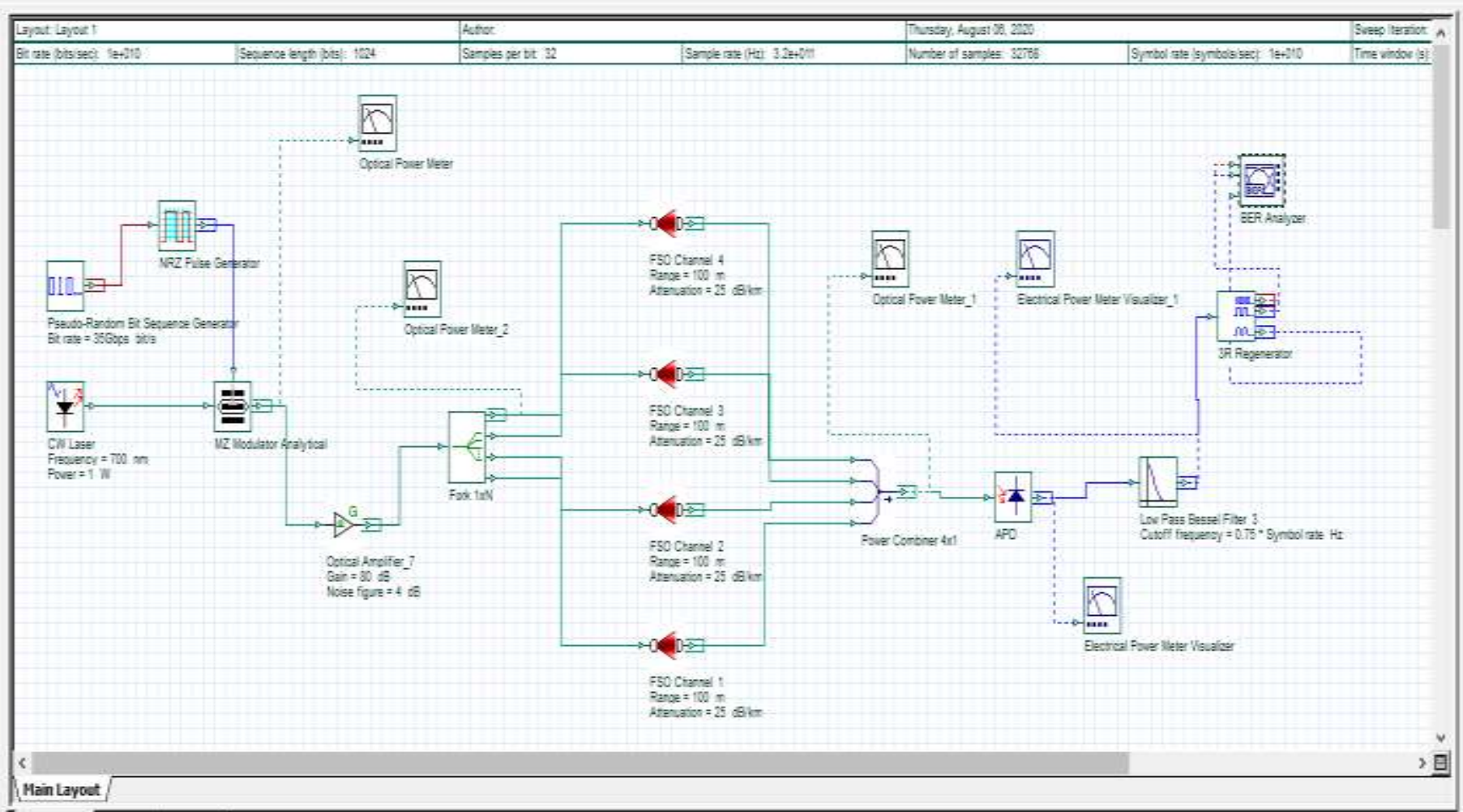

Fig.3: The layout of MIMO (Multiple Input Multiple Output) Visible light communication transceivers (4x4 MIMO) using 4 FSO channels.

\section{Simulation PARAMETERS AND RESUlts}

The $\mathrm{Q}$ factor is a degree of how noisy a pulse is, this is for analysis commitments. The oscilloscope will normally produce a report that shows the $\mathrm{Q}$ factor number. A larger number of $\mathrm{Q}$ factor in the result means that the pulse is relatively free from noise. The eye diagram of BER analyzer shows that the maximum Q factor of MIMO is relatively large when compared to SISO. The BER analyzer output for SISO and MIMO is as shown in Fig.6 and Fig.7 respectively. That is 10381.4 and 10902.6 is the maximum $\mathrm{Q}$ factor for SISO and MIMO system respectively. Hence the pulse is relatively free from noise indicating better performance. As the distance between transmitter and receiver is 100 meters, this can be implemented in SISO form and also as MIMO form for larger applications.

Almost all the parameters considered for SISO and MIMO are same except optical amplifier gain. As the number of transmitters and receivers are more in MIMO and there are four FSO channels. The optical amplifier gain is multiplied by four. The simulation parameters and their values are listed below in tabular form. Table 1 show the parameters which are considered for the simulation purpose.

Table 1 Simulation Parameters

\begin{tabular}{|l|l|l|}
\hline \multicolumn{1}{|c|}{ Component } & \multicolumn{1}{|c|}{ Parameter } & \multicolumn{1}{c|}{ value } \\
\hline PR bit sequence generator & Bit rate & $35 \mathrm{Gbps}$ \\
\hline CW laser & $\begin{array}{l}\text { Frequency } \\
\text { Power }\end{array}$ & $\begin{array}{l}700 \mathrm{~nm} \\
1 \mathrm{Watt}\end{array}$ \\
\hline Optical Amplifier & Gain & $\begin{array}{l}20 \mathrm{~dB}(\mathrm{SISO}) \\
80 \mathrm{~dB}(\mathrm{MIMO}) \\
\end{array}$ \\
& Noise figure & $4 \mathrm{~dB}$ \\
\hline FSO channel & Range & $100 \mathrm{~m}$ \\
& Attenuation & $25 \mathrm{~dB} / \mathrm{Km}$ \\
\hline
\end{tabular}




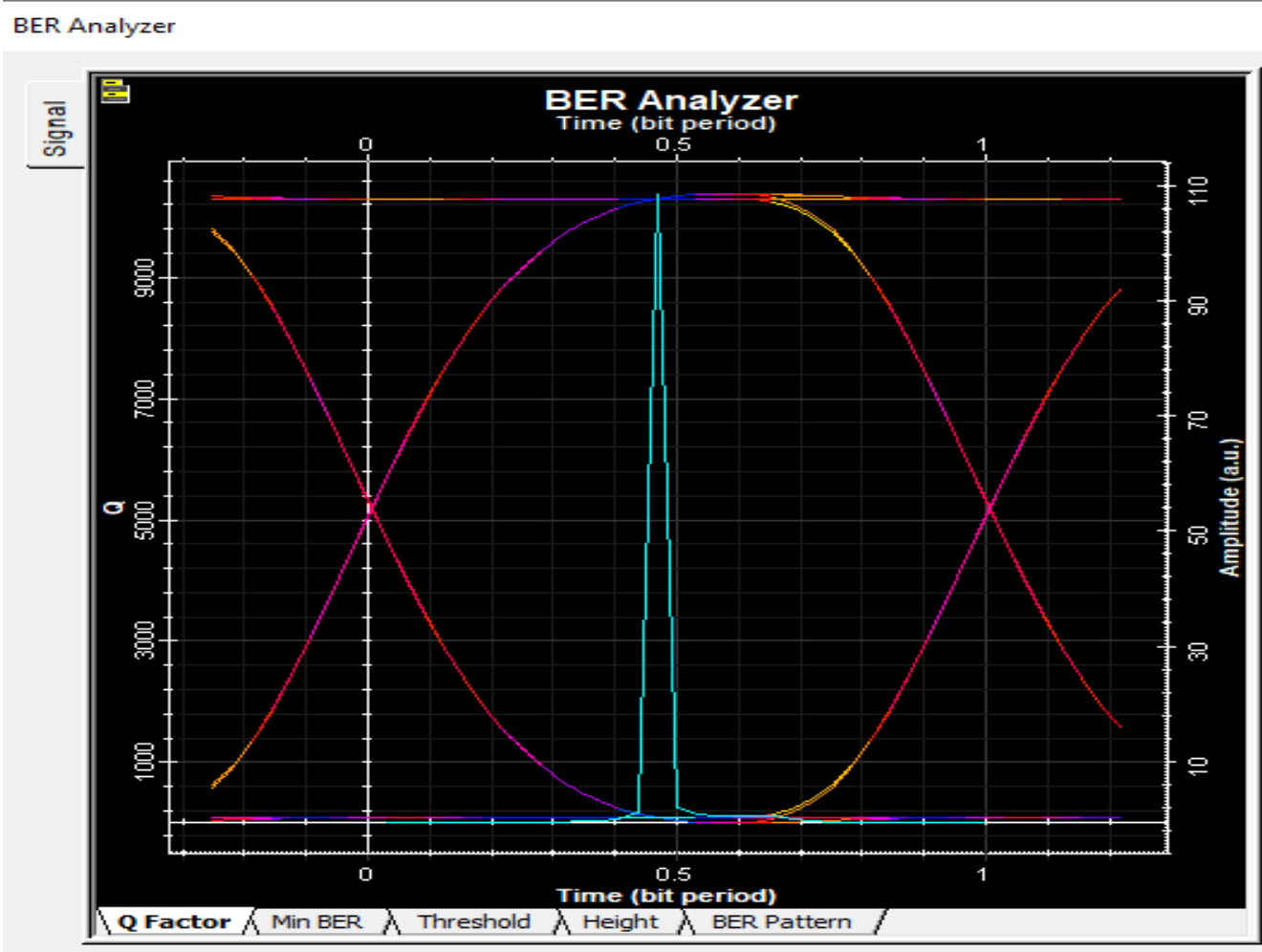

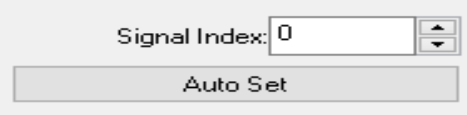

$\square$ Show Eye Diagram

\begin{tabular}{|l|r|}
\multicolumn{1}{|c|}{ Analysis } & \\
\hline Max. Q Factor & 10381.4 \\
\hline Min. BER & 0 \\
\hline Eye Height & 107.724 \\
\hline Threshold & 13.28 \\
\hline Decision Inst. & 0.53125 \\
\hline
\end{tabular}

$\square$ Invert Colors

$\checkmark$ Color Grade

Patterns

Calculate Patterns
Patterns |
\begin{tabular}{|l|l|}
\hline Pattern 1 & $1 e-012$ \\
\hline & Pattern 2 \\
\hline Pattern 3 & $1 e-011$ \\
\hline Pattern 4 & $1 e-010$ \\
\hline Pattern 5 & $1 e-009$ \\
\hline
\end{tabular}

Fig.6: BER analyzer output of SISO (Single Input Single Output) Visible light communication transceivers using FSO channel.

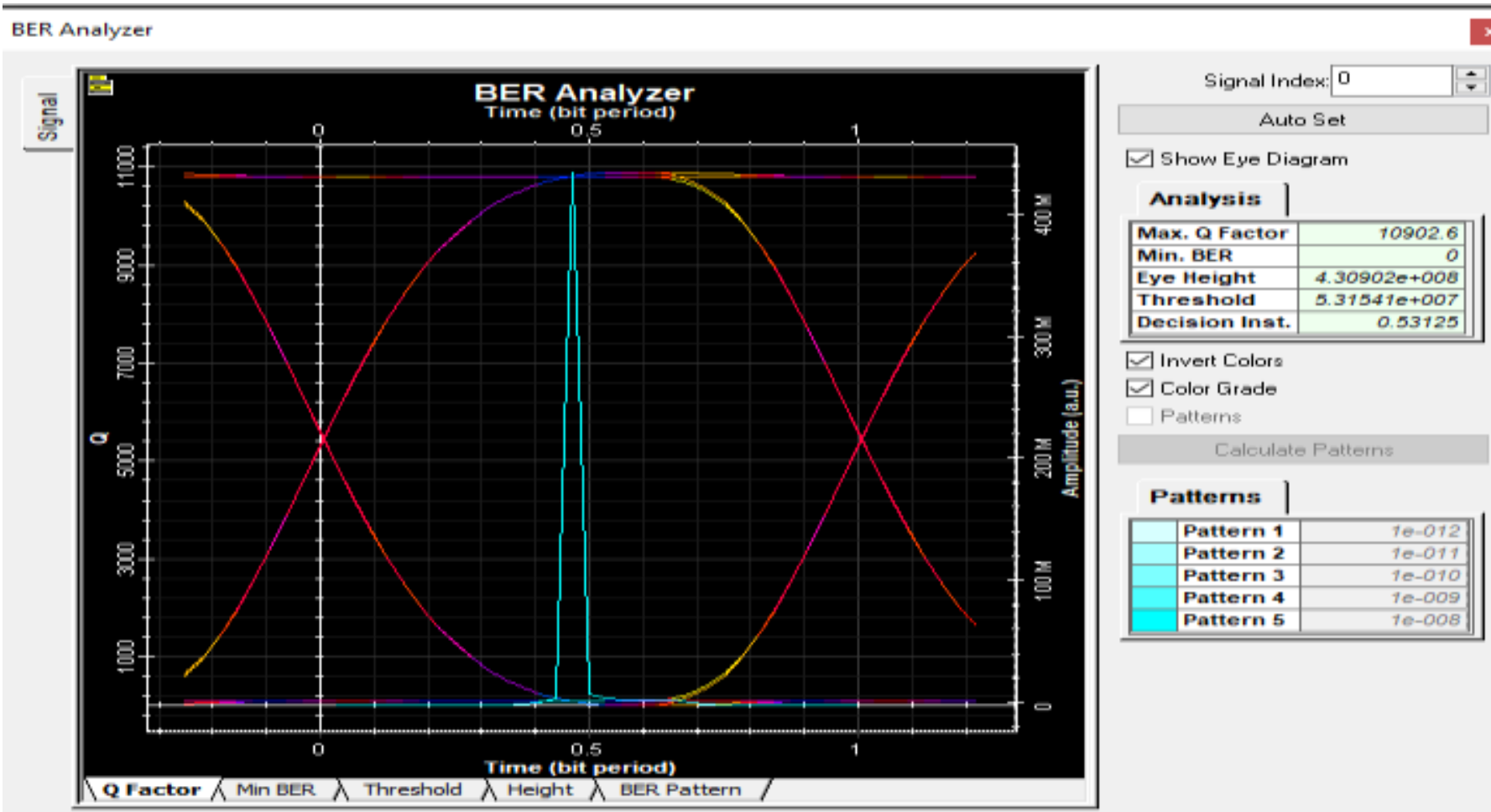

Fig.7: The BER analyzer output of MIMO (Multiple Input Multiple Output) Visible light communication transceivers (4x4 MIMO) using 4 FSO channels

\section{CONCLUSION}

This paper is all about data transmission through visible light. Using the visible light spectrum, data transmission from one PC to another is successfully done for smaller distance. For longer distance communication, simulations are done by transmitting data at a bit rate of 35Gbps. Both SISO and MIMO VLC systems are designed and simulated. The distance is set to 100 meters and is 
sufficient for indoor VLC system. In future work, FSO channels can be implemented for outdoor communication purpose with distance in terms of kilometers. But, in outdoor VLC, atmospheric conditions should be taken into consideration for speedy communication.

\section{REFERENCES}

[1] S. Das, A. Chakraborty, D. Chakraborty and S. Moshat, "PC to PC data transmission using visible light communication," 2017 International Conference on Computer Communication and Informatics (ICCCI), Coimbatore, 2017, pp. 1-5, doi: 10.1109/ICCCI.2017.8117767.

[2] Mr. Yogesh Chawan, Mr. Ramchandra Gaurav, "Data Transfer using Visible Light Communication”, 2017 - Volume 4 Issue 2 January 2017, IARJSET

[3] Zashi P. Choudhari and Satish R. Devane. "High sensitivity universal Lifi receiver for enhance data communication". Online International Conference on Green Engineering and Technologies (IC-GET), 2016, Mumbai, INDIA.

[4] M.Lotfy Rabe, Mohammad I Gabar, Tarek Husny, "Data Transmission via Visible Light Communication (VLC) Technique", Volume 5 Issue 9, September 2016, IJIRSET (ISSN: 2319-8753)

[5] Prashant D. Nehare, Mayur Purohit, Chaitali S. Somase, Piyush Sarode, "Transmission of Data through [Li-Fi] Light Fidelity From One PC to another PC", IRJET, Volume 4 Issue 4, Apr-2017.

[6] Akash Gupta et.al." Cascaded FSO-VLC Communication System". IEEE Wireless Communications, 2017.

[7] Chetna Verma and Chetan Selwal "Visible light communication system (VLC) Using Diversity Technique with 4 QAM OFDM FSO Link", IEEE Xplore, 2018.

[8] Hammed G. Olanrewaju et.al. "Pairwise Coding for MIMO-OFDM Visible Light Communication". IEEE TRANSACTIONS ON WIRELESS COMMUNICATIONS, 2019.

[9] Fabian Harendran Jesuthasan et.al." Implementation and Performance Evaluation of a MIMO-VLC System for Data Transmissions". IEEE, 2019.

[10] https://www.arduino.cc/en/Main/Boards

[11] https://www.prophotonix.com/resources/technical-overviews/led-array-methods/

[12] http://henrysbench.capnfatz.com/henrys-bench/arduino-output-devices/tip122-arduinorelay-tutorial/

[13] Liang K., Chow C.W., Liu Y. "RGB visible light communication using mobile-phone camera and multi-input multi-output". Opt. Express.2016.

[14]Zhitong Huang et.al." Hybrid Optical Wireless Network for Future SAGO-Integrated Communication Based on FSO/VLC Heterogeneous Interconnection". IEEE Photonics Journal, 2017.

[15] Milica Petkovic.” Outage Probability Analysis of RF/FSO-VLC Communication Relaying System”. IEEE.

[16] https://en.wikipedia.org/wiki/Avalanche_photodiode

[17] https://optiwave.com/forums/topic/3r-regenerator/ 